\title{
Ontological framework based on contextual mediation for collaborative information retrieval
}

\author{
Jason J. Jung
}

Published online: 3 February 2007

(C) Springer Science + Business Media, LLC 2007

The Publisher regrets that this article was placed in the incorrect issue, volume 10:1, January 2007. It should have appeared in this special issue, Contextual Information Retrieval Systems, volume 10:2, March 2007. 